\title{
Durvalumab-Induced Diffuse Alveolar Hemorrhage: An Autopsy Case Report
}

\author{
Kensuke Kanaoka ${ }^{a}$ Saori lkebe ${ }^{a}$ Shouichi Ihara ${ }^{a}$ Hiromi Tsuji ${ }^{b}$ \\ Hironao Yasuokab Seigo Minami ${ }^{a}$ \\ aDepartment of Respiratory Medicine, Osaka Police Hospital, Osaka, Japan; \\ bepartment of Pathology, Osaka Police Hospital, Osaka, Japan
}

\section{Keywords}

Immune checkpoint inhibitors · Durvalumab · Diffuse alveolar hemorrhage · Bronchoalveolar lavage fluid $\cdot$ Immune-related adverse events

\begin{abstract}
Durvalumab, a programmed cell death ligand 1 inhibitor, induces various immune-related adverse events (irAEs), including lung injury. However, diffuse alveolar hemorrhage (DAH) is a rare type of lung injury due to immune checkpoint inhibitors. A 76-year-old man with c-stage IIIA squamous cell carcinoma of the lung received maintenance durvalumab therapy after chemoradiotherapy. He developed dyspnea and malaise after 11 cycles of durvalumab. Chest computed tomography showed rapidly spreading bilateral ground-glass opacity in the lungs. We diagnosed DAH by hemosiderin-laden macrophages in bloody bronchoalveolar lavage fluid. Despite mechanical ventilation, steroids, and cyclophosphamide, he died of respiratory failure. The autopsy revealed that fresh and old bleeding areas coexisted, and neither pulmonary vasculitis nor diffuse alveolar damage was detected microscopically. Furthermore, CD3+ and CD8+ lymphocytes were observed in the lung interstitium, whereas CD20+ and CD4+ lymphocytes were scarcely detected. We report the first case of durvalumab-induced DAH. We should be alert to irAEs with DAH as a potential differential diagnosis of lung injury during durvalumab treatment.

(c) 2020 The Author(s).

Published by S. Karger AG, Basel
\end{abstract}

\section{Introduction}

Durvalumab maintenance therapy after chemoradiotherapy improves progression-free and overall survival for patients with inoperable stage III non-small cell lung cancer $[1,2]$. Among various immune-related adverse events (irAEs) induced by programmed cell death 


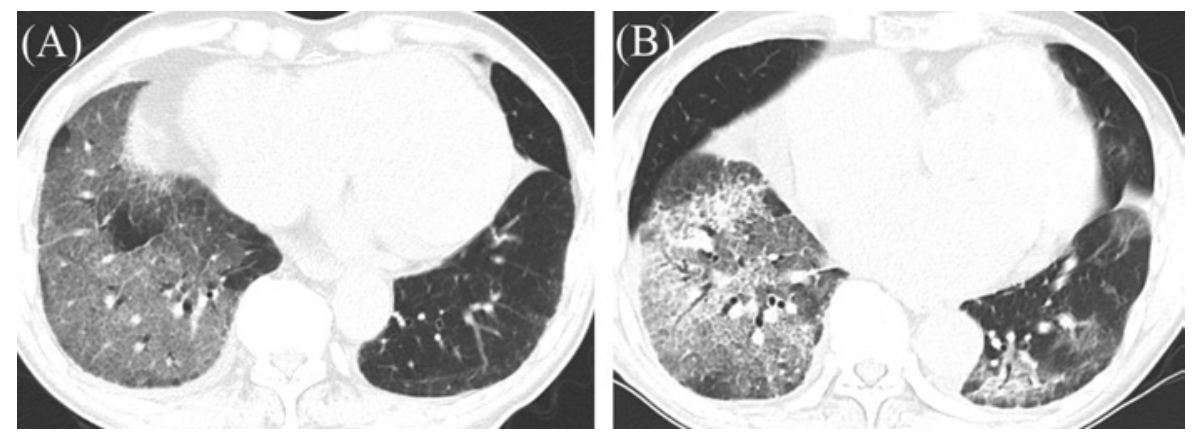

Fig. 1. Chest CT images on the first hospital day showed ground-glass opacity in the bilateral lower lobes, extending beyond the irradiated areas $(\mathbf{A})$, and on the tenth hospital day showed ground-grass opacity spread to the entire left lung (B).

protein 1 (PD-1)/programmed cell death ligand 1 (PD-L1) inhibitors, lung injury is one of the most serious adverse events [3]. In the PACIFIC trial, lung injury occurred in $33.9 \%$ of patients treated with durvalumab, and was the most frequent grade 5 irAE [2]. According to a study on lung injury in patients treated with PD-1/PD-L1 inhibitors, the most common pathological feature was cellular interstitial pneumonitis, and the second most common was organizing pneumonia [4]. However, to the best of our knowledge, diffuse alveolar hemorrhage (DAH) has never been reported as a lung injury induced by durvalumab. It is difficult to distinguish DAH from other types of lung injury based on clinical presentation and radiographic examination [5]. Hemoptysis is a specific symptom of DAH, but it is initially absent in 33\% of patients with DAH [6]. Radiologic findings of bilateral and central ground-glass opacities (GGOs) are nonspecific and can change throughout the course of the disease [5, 7]. In clinical practice, DAH is often diagnosed by bronchoalveolar lavage (BAL). Bloody BAL fluid (BALF), intraalveolar red blood cells, and hemosiderin-laden macrophages indicate a diagnosis of DAH [6, 7]. Herein, we report an autopsy case of durvalumab-induced DAH.

\section{Case Report}

A 76-year-old man was diagnosed with squamous cell carcinoma of the lung (cT2aN2M0, c-stage IIIA). He regularly took edoxaban for atrial fibrillation, and he received chemoradiotherapy ( 2 Gy $\times 33$ fractions with weekly carboplatin and weekly paclitaxel). Nine days after the end of thoracic radiation, he developed a fever of $38.6^{\circ} \mathrm{C}$. Chest computed tomography (CT) showed a new GGO in the irradiation area. We suspected radiation pneumonitis and then initiated oral prednisolone therapy (initial dose: $1 \mathrm{mg} / \mathrm{kg} /$ day). We gradually reduced the dose and then discontinued prednisolone for 6 months. When the dose of prednisolone had decreased to $10 \mathrm{mg} /$ day, 41 days after the thoracic radiotherapy, we initiated durvalumab maintenance treatment (10 mg/kg, bi-weekly).

After 11 cycles of durvalumab therapy, 3 weeks after the discontinuation of prednisolone, he developed dyspnea and malaise. His body temperature was $37.3^{\circ} \mathrm{C}$, heart rate was 75 beats per minute, blood pressure was $111 / 81 \mathrm{~mm} \mathrm{Hg}$, and oxygen saturation on room air was $96 \%$. Chest CT showed new GGOs in the bilateral lower lobes, extending beyond the irradiated areas (Fig. 1A). Laboratory blood tests exhibited an increase in C-reactive protein $(8.24 \mathrm{mg} / \mathrm{dL})$ and KL-6 (515 U/mL). He was hospitalized and underwent fiberoptic bronchoscopy on hospital day 2. The BALF from the right $B_{8}$ gradually became bloody (Fig. $2 A$ ), and revealed a cell count 

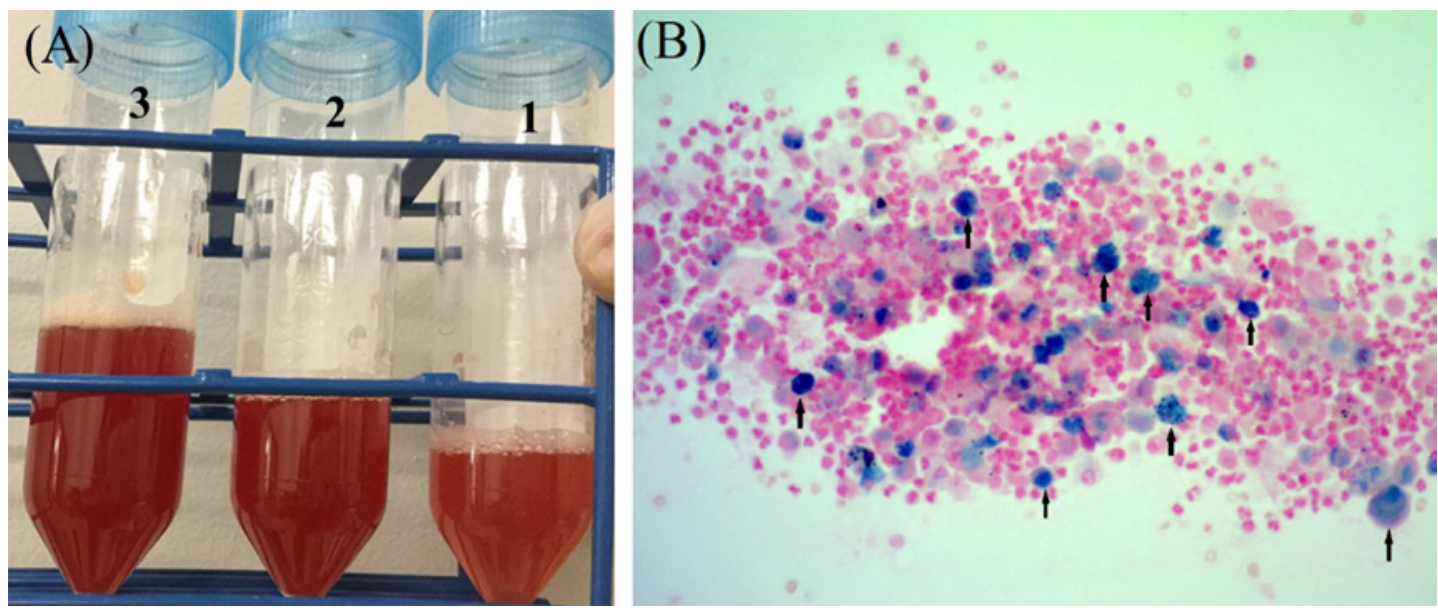

Fig. 2. Macroscopic (A) and microscopic (B) findings of bronchoalveolar lavage fluid from the right B8 on the second hospital day. B Berlin blue staining. $\times 100$. Arrows indicate hemosiderin-laden macrophages.
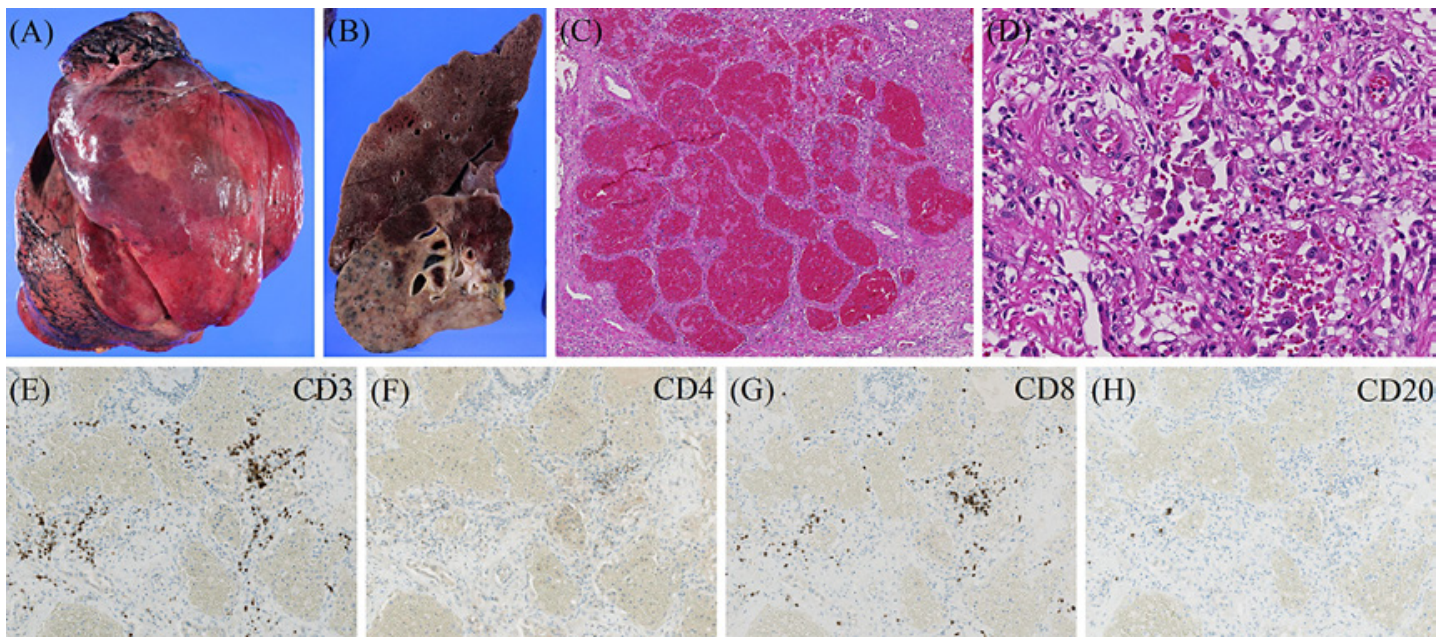

Fig. 3. Autopsy findings. Microscopic view of autopsied right lung before formalin fixation (A) and after formalin fixation (B). Microscopic finding of alveolar spaces filled with red blood cells in a fresh bleeding area of the lung (C) (hematoxylin and eosin stain; $\times 40$ ) and hemosiderin-laden macrophages in an old bleeding area of the lung (D) (hematoxylin and eosin stain; $\times 200$ ). Immunostaining $(\times 100)$ for CD3 (E), CD4 (F), CD8 (G), and CD20 (H); predominantly CD3+ and CD8+ lymphocytes infiltrated the lung interstitium (E, G), while there were few CD20+ and CD4+ lymphocytes (F, H).

of $13.4 \times 10^{5}$ cells $/ \mathrm{mL}$ with $70 \%$ neutrophils, $10.5 \%$ lymphocytes, $11 \%$ eosinophils, and $8.5 \%$ macrophages. Despite immediate discontinuation of edoxaban and administration of ciprofloxacin, his symptoms and respiratory conditions rapidly deteriorated. Thereafter, although there was a negative bacterial culture in the BALF, we detected hemosiderin-laden macrophages (Fig. 2B). Myeloperoxidase ANCA (antineutrophil cytoplasmic antibody), proteinase-3 ANCA, and anti-glomerular basement membrane antibody were all negative. Thus, we suspected durvalumab-induced DAH. On hospital day 7, we initiated oral prednisolone (1 mg/ $\mathrm{kg} /$ day). Chest CT on hospital day 10 showed an expansion of GGOs to the left lung (Fig. 1B). A methylprednisolone pulse $(1,000 \mathrm{mg} /$ day $)$ was administered for 3 consecutive days, followed 
Kanaoka et al.: Durvalumab-Induced Diffuse Alveolar Hemorrhage

Table 1. Review of diffuse alveolar hemorrhage caused by immune checkpoint inhibitors in the English literature

\begin{tabular}{|c|c|c|c|c|}
\hline & Takahashi et al. [8], 2018 & Sugano et al. [9], 2018 & Ikeda et al. [10], 2018 & Our report \\
\hline Cancer (histology) & Lung (Ad) & Lung (Ad) & Melanoma & Lung (sq) \\
\hline ICIs & Nivolumab & Pembrolizumab & Nivolumab & Durvalumab \\
\hline TPS & Not described & $50-74 \%$ & Not described & $10-20 \%$ \\
\hline Patient's age, years (sex) & $74(\mathrm{M})$ & $67(F)$ & $41(\mathrm{~F})$ & $76(\mathrm{M})$ \\
\hline Symptoms & Hematuria, hemoptysis & No symptoms & $\begin{array}{l}\text { Bloody sputum, } \\
\text { respiratory distress }\end{array}$ & Dyspnea, malaise \\
\hline DAH onset timing & 16 weeks (8 cycles) & 4 cycles & 3 months & 5 months ( 11 cycles) \\
\hline CT images & $\begin{array}{l}\text { GGO (unilateral and } \\
\text { not central) effusion }\end{array}$ & $\begin{array}{l}\text { GGO and consolidation } \\
\text { (unilateral and not } \\
\text { central) }\end{array}$ & $\begin{array}{l}\text { GGO (bilateral and not } \\
\text { central) }\end{array}$ & $\begin{array}{l}\text { GGO (bilateral and } \\
\text { not central) }\end{array}$ \\
\hline $\mathrm{CRP}, \mathrm{mg} / \mathrm{dL}$ & Not described & 4.7 & 11.85 & 8.24 \\
\hline Diagnosis of DAH & Autopsy & BALF & BALF & BALF \\
\hline Lymphocytes in BALF & Not described & $45.0 \%$ & $32.2 \%$ & $10.5 \%$ \\
\hline Treatment & $\begin{array}{l}\text { mPSL pulse } \\
\text { Plasma exchange }\end{array}$ & Oral PSL (1 mg/kg/day) & mPSL pulse & $\begin{array}{l}\text { mPSL pulse } \\
\text { Cyclophosphamide }\end{array}$ \\
\hline Outcome & Died (35 days) & Improved & Improved & Died (50 days) \\
\hline Note & Goodpasture's disease & & Pseudoprogression & \\
\hline
\end{tabular}

Ad, adenocarcinoma; BALF, bronchoalveolar lavage fluid; CRP, C-reactive protein; DAH, diffuse alveolar hemorrhage; F, female; GGO, ground-glass opacity; ICI, immune checkpoint inhibitor; M, Male; mPSL, methylprednisolone; PSL, prednisolone; Sq, squamous cell carcinoma; TPS, tumor proportion score.

by oral prednisolone ( $1 \mathrm{mg} / \mathrm{kg} /$ day). On hospital day 14 , due to his deteriorated respiratory condition, we introduced invasive mechanical ventilation with endotracheal intubation. On hospital day 15, we administered cyclophosphamide $\left(750 \mathrm{mg} / \mathrm{m}^{2}\right)$. The second BALF from the left $\mathrm{B}_{5}$ and $\mathrm{B}_{9}$ on hospital day 24 were both bloody and contained hemosiderin-laden macrophages. Due to exacerbated respiratory failure, the patient died on day 50.

We performed an autopsy $14 \mathrm{~h}$ after his death. In the macroscopically bloody lung areas with decreased aeration (Fig. 3A, B), the alveolar spaces were microscopically filled with red blood cells, which suggested fresh bleeding (Fig. 3C). In the macroscopically white lung areas, hemosiderin-laden macrophages were still present, which suggested old bleeding (Fig. 3D). Almost all parts of the lungs exhibited one of these two histological findings. Neither pulmonary vasculitis nor diffuse alveolar damage was detected in the autopsy. Immunohistochemical analyses showed that $\mathrm{CD} 3$-positive (CD3+) and CD8+ lymphocytes were present in the lung interstitium, but not around capillaries. On the other hand, CD20+ and CD4+ lymphocytes were scarcely observed (Fig. 3E-H).

\section{Discussion}

To our knowledge, this was the first case of durvalumab-induced DAH, and the second case of an autopsy after an irAE of DAH. The clinically important finding is that DAH is a possible irAE due to durvalumab. There have been only 3 case reports of DAH induced by 
PD-1 inhibitors (Table 1) [8-10]. Except for an autopsy case of DAH due to nivolumab-induced Goodpasture's disease, the etiology of the other 2 DAH cases is unknown. In these 2 cases, unlike in our case, the responses to steroids were good. A higher proportion of lymphocytes in BALF might be associated with better outcomes. In our case, we did not suspect DAH until BALF, because the patient did not complain of hemoptysis and the radiologic findings were not typical of DAH. We are afraid of underdiagnosed immune checkpoint inhibitor (ICI)induced DAH when BAL is not performed; thus, we should not hesitate to perform BAL when we suspect lung injury as an irAE.

The pathologically important finding was that bland pulmonary hemorrhage was the histopathological feature of our DAH. There are three pathological patterns that can induce DAH: pulmonary capillaritis, diffuse alveolar damage, and bland pulmonary hemorrhage [6]. Except for the autopsy case of Goodpasture's disease, neither an autopsy nor a lung biopsy was performed in the other 2 DAH cases [8-10]. Thus, the pathological mechanism underlying DAH as an irAE has remained unknown. Our autopsy showed a bland pulmonary hemorrhage pattern. Although anticoagulant drugs, including edoxaban, can induce bland pulmonary hemorrhage, in previous case reports the hemorrhage disappeared immediately after the drugs were stopped [11]. In our case, there was still fresh bleeding at death, which means that the bleeding continued 7 weeks after the discontinuation of edoxaban. Therefore, edoxaban did not cause DAH in our case. There were 2 cases in which lung injury as an irAE had occurred 6 and 8 months after stopping ICIs [12]. These 2 cases do not contradict our finding that DAH continued for more than 10 weeks after discontinuation of durvalumab. Thus, we should be particularly alert to lung injury as an irAE for several months after ICI therapy.

Interestingly, immunostaining revealed that the predominant infiltrating lymphocytes in the lung interstitium were exclusively CD3+ and CD8+ cells, and there were few CD20+ and CD4+ cells. Our immunostaining patterns in the lungs were similar to those of an irAE of liver injury, which are characterized by the infiltration of predominantly CD3+ and CD8+ lymphocytes, with fewer CD20+ or CD4+ lymphocytes detected [13]. Furthermore, the CD20+/CD3+ and CD4+/CD8+ ratios were significantly lower than those in autoimmune or drug-induced liver injuries. However, it is still unclear whether CD3+ and CD8+ lymphocytes increase or not in an irAE of lung injury. Immunostaining for CD4 and CD8 might be useful for distinguishing lung injury as an irAE from other types of lung injuries.

In conclusion, we report the first case of durvalumab-induced DAH. We should be alert to an irAE of DAH as a potential differential diagnosis of lung injury during durvalumab treatment.

\section{Acknowledgements}

We thank Dr. Kazunori Moriizumi, Dr. Kanako Nishimatsu, and Dr. Hideyasu Okada (the Department of Respiratory Medicine, Osaka Police Hospital) for their contributions to the care and management of this patient.

\section{Statement of Ethics}

The patient's son has given informed consent to publish this case including publication of images. 
Kanaoka et al.: Durvalumab-Induced Diffuse Alveolar Hemorrhage

\section{Disclosure Statement}

All authors declare no potential conflicts of interest related to the publication of this case report.

\section{Funding Sources}

We declare that the authors received no funds for this research.

\section{Author Contributions}

K. Kanaoka, S. Ikebe, S. Ihara, and S. Minami were involved in treatment of this patient. H. Tsuji and H. Yasuoka performed the pathological diagnosis of the tumor and DAH. K. Kanaoka drafted the report. All authors read and critically reviewed the report. All authors approved the final submitted version.

\section{References}

1 Antonia SJ, Villegas A, Daniel D, Vicente D, Murakami S, Hui R, et al. Overall survival with durvalumab after chemoradiotherapy in stage III NSCLC. N Engl J Med. 2018;379(24):2342-50.

2 Antonia SJ, Villegas A, Daniel D, Vicente D, Murakami S, Hui R, et al. Durvalumab after chemoradiotherapy in stage III non-small-cell lung cancer. N Engl J Med. 2017;377(20):1919-29.

3 Wang DY, Salem JE, Cohen JV, Chandra S, Menzer C, Ye F, et al. Fatal toxic effects associated with immune checkpoint inhibitors: a systematic review and meta-analysis. JAMA Oncol. 2018;4(12):1721-8.

4 Naidoo J, Wang X, Woo KM, Iyriboz T, Halpenny D, Cunningham J, et al. Pneumonitis in patients treated with anti-programmed death-1/programmed death ligand 1 therapy. J Clin Oncol. 2017;35(7):709-17.

5 Lichtenberger JP, Digumarthy SR, Abbott GF, Shepard JA, Sharma A. Diffuse pulmonary hemorrhage: clues to the diagnosis. Curr Probl Diagn Radiol. 2014;43(3):128-39.

6 Lara AR, Schwarz MI. Diffuse alveolar hemorrhage. Chest. 2010;137(5):1164-71.

7 Krause ML, Cartin-Ceba R, Specks U, Peikert T. Update on diffuse alveolar hemorrhage and pulmonary vasculitis. Immunol Allergy Clin North Am. 2012;32(4):587-600.

8 Takahashi N, Tsuji K, Tamiya H, Shinohara T, Kuroda N, Takeuchi E. Goodpasture's disease in a patient with advanced lung cancer treated with nivolumab: an autopsy case report. Lung Cancer. 2018;122:22-4.

9 Sugano T, Seike M, Noro R, Kaburaki S, Tozuka T, Takahashi A, et al. A case of interstitial lung disease with alveolar hemorrhage induced by pembrolizumab. Onco Targets Ther. 2018;11:5879-83.

10 Ikeda T, Yamaguchi H, Dotsu Y, Taniguchi H, Gyoutoku H, Senju H, et al. Diffuse alveolar hemorrhage with pseudoprogression during nivolumab therapy in a patient with malignant melanoma. Thorac Cancer. 2018; 9(11):1522-4.

11 Nitta K, Imamura H, Yashio A, Kashima S, Mochizuki K. Diffuse alveolar hemorrhage associated with edoxaban therapy. Case Rep Crit Care. 2016;2016:7938062.

12 Couey MA, Bell RB, Patel AA, Romba MC, Crittenden MR, Curti BD, et al. Delayed immune-related events (DIRE) after discontinuation of immunotherapy: diagnostic hazard of autoimmunity at a distance. J Immunother Cancer. 2019;7(1):165-11.

13 Zen Y,Yeh MM. Hepatotoxicity of immune checkpoint inhibitors: a histology study of seven cases in comparison with autoimmune hepatitis and idiosyncratic drug-induced liver injury. Mod Pathol. 2018;31(6):965-73. 